\title{
Effect of white noise on bar-press rate in rats
}

ALICE KUHN SCHWARTZ

HOFSTRA UNIVERSITY

White noise was paired with food reinforcement for leverpress. Each of four groups received one intensity of sound; the fifth group was given no sound. Results indicate that response rate is a positive function of sound intensity. Extinction data show that response-contingent sound in conjunction with food reinforcement exerts its influence on performance rather than learning. Similarities between re inforcement-complex intensity and $S^{D}$ intensity were pointed out. It was suggested that both are subject to the laws of stimulus intensity dynamism.

Various intensities of white noise have been shown to suppress preceding behavior by their onset or to reinforce preceding behavior by their termination (Barnes \& Kish, 1957; Barry \& Harrison, 1957; Bolles \& Seelbach, 1964; Campbell, 1955; Harrison \& Abelson, 1959). At the same time, there is evidence that comparable intensities when used as conditioned stimuli have produced response strength in positive relationship to the intensity employed (Barnes, 1956; Beck, 1963; Gray, 1965; Meyers, 1962; Walker, 1960; Zener, 1949).

In view of the seemingly opposite effects obtained with signal sound and response produced sound, it was deemed advisable to test the effect of noise in yet another situation, that is, in conjunction with food reinforcement. The present investigation was designed to determine whether response-contingent sound paired with food reinforcement would serve to depress bar-press rate in the manner of other response produced sound or to increase bar-press rate as in the case of CS sound. A second purpose of the present study was to ascertain whether sound administered in this way exerts its effect on learning or performance.

Subjects

The Ss were 30 male rats of the Fisher strain, approximately 120 days old at the beginning of the experiment. They had been handled but were experimentally naive. All Ss were $23 \mathrm{hr}$. food deprived in the test situation. Apparatus

Preliminary bar-press training was given in four Skinner boxes. After acquisition of the bar-press response, Ss were placed in either of two metal chests, each having 11 in. $x 20$ in. inside measurements, a hinged wood front door, opening downward, and a one-way mirror to permit observation of S. Each chest contained two compartments. The left compartment measured 11 in. deep, $11 \mathrm{in}$, high, and 9 in. wide. It contained a grid floor, a food cup, and a 7-W houselight. The second compartment contained a Gerbrands feeder. A $28-\mathrm{V}$ relay operated the counter and feeder and the sound when it was switched on. The relay was activated by bar-depression. Power for each chamber was supplied by a $28-\mathrm{V}$ dc power supply unit. The sound sources were one Lehigh Valley white noise generator (Model 1524) and one Grason-Stadler white noise generator (Model 4558). Sound was measured by a General Radio sound level meter (Model 1551C). The meter was calibrated by a General Radio sound level calibrator (Model 1307A) and a General Radio transistor oscillator (Model 1307C). Procedure

Ss were maintained on a 23-hr. deprivation schedule for 10 days prior to experimentation. Five 30-min. barpress acquisition sessions were given in four laboratory Skinner boxes. Reinforcement consisted of standard reinforcement pellets weighing $45 \mathrm{mg}$ each. The Ss were then tested in either of the metal sound attenuating boxes, the same $S$ always being tested in the same box. The two test rooms were distant from each other. Seven additional 30-min. sessions were given. Because experimental sessions started at 8:30 A.M. and continued until 5:00 P.M. each day, a comparison of bar-press rates between morning, afternoon, and early evening Ss was made by means of Chi-square. The results showed time of day as a source of variation in bar-press rate to be nonsignificant.

The Ss were placed in five matched groups and on new feeding schedules that would bring each $S$ to the test situation $23 \mathrm{hr}$. food deprived. On the fourth day following the constitution of the new groups, and thereafter, in addition to food reinforcement, each group was given a blast of white noise for the duration of each bar-press. White noise intensities (re: .0002 dyne $/ \mathrm{cm}^{2}$ ) were as follows: Group 1, $110 \mathrm{~dB}$; Group 2, $95 \mathrm{~dB}$; Group 3, $80 \mathrm{~dB}$; Group 4, $65 \mathrm{~dB}$; and Group 5, no sound. Thirty-min. test sessions were given to each $S$ on 12 consecutive days.

At the end of 12 days, each experimental group was subdivided into two groups, each containing one high, one medium, and one low responder for that group. For one subgroup, the newly established control group, conventional extinction procedures were employed. For the experimental subgroup, conventional extinction procedures were supplemented by noise onset contingent

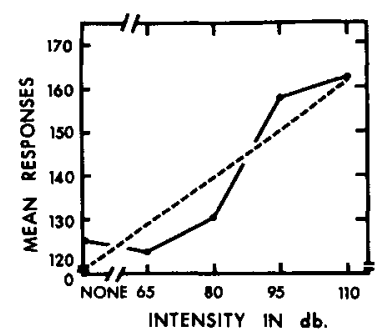

Fig. 1. Mean response rate for five groups on the 12th day of sound stimulation. 


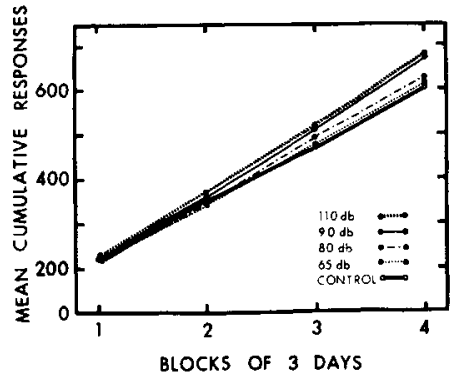

Fig. 2. Mean cumulative responses during 12 days of sound stimulation.

upon each bar-press. Three 30-min. extinction sessions were administered on three consecutive days.

Results and Discussion

Clearly, sound stimulation raises bar-press rate under the present conditions. Figure 1 shows the mean response rate for the five groups on the twelfth day of sound stimulation.

A Hartley test for homogeneity of variance was applied to sound stimulation scores resulting in an $F$ max of 7.16. The hypothesis of homogeneity of variance could not be rejected. Scores during the 12 days of sound stimulation were subjected to two-way analysis of variance. No significant variation with intensity was shown, $\mathbf{F}=$ 1.45, $\mathrm{df}=4 / 25, \mathrm{p}>.05$. A significant effect of days was disclosed, $F=13.37, \mathrm{df}=3 / 75, \mathrm{p}<.01$. A significant interaction variance was obtained, $F=2.44, \mathrm{df}=12 / 75$, $\mathrm{p}<.05$.

Cumulative mean responses during sound stimulation are plotted in Fig. 2. Inspection of Fig. 2 indicates that responding is a positive function of the intensity of the sound stimulus.

Extinction data were submitted to analysis of variance with resulting $\mathbf{F}$ values which proved to be nonsignificant.

The increase in rate during sound stimulation over training rate without sound shows clearly that noise, in conjunction with food, increases bar-press rate. Moreover, response rate stands in direct relationship to sound intensity for each of the four values employed, the lowest value in turn producing a higher cumulative mean than that of the control group. Inspection of curves indicates that rate of bar-pressing is an increasing function of noise intensity. The graphical evidence that differential rates of responding emerged as a function of stimulus intensity during the course of training is strengthened by the significant interaction between days and intensity. The lack of significance in variance due to intensity is disappointing. However, when there is a relatively slight margin between base rate and physio- logical limit, the situation may preclude the achievement of statistical significance. Failure of analysis of variance to show intensity as a significant source of variation suggests that either the main effect was not strong enough or the test not sensitive enough to disclose differences which are manifest upon inspection.

The data show more similarity to signal stimuli data than to effects obtained with simple response-produced sound. The fact that response strength increases in relation to CS intensity has been attributed to the Pavlovian "law of strength" or its counterpart, Hull's "stimulus intensity dynamism." It may be that the laws of "stimulus intensity dynamism" ' apply to a reinforcing stimulus as well as to an $\mathrm{S}^{\mathrm{D}}$.

Extinction data showed performance rather than habit strength to be affected. This is entirely consonant with results of Kessen (1953) and Walker (1960). Kessen argued that the strength of the conditioned stimulus affects reaction potential rather than learning. This additional similarity serves to strengthen the relationship between CS and reinforcement complex, and further suggests that both are governed by the same laws.

\section{References}

Bames, G. W. Conditioned stimulus intensity and temporal factors in spaced-trial classical conditioning. J. exp. Psychol., 1956. 51, 192-198.

Bames, G. W., \& Kish, G. B. Reinforcing properties of termination of intense auditory stimulation. $J$. comp. physiol. Psychol., 1956, 50, 40-43.

Barry, J., Jr., \& Harrison, J. M. Relation between stimulus intensity and strength of escape responding. Psychol. Rep., 1957, 3, 3-8.

Beck, S. B. Eyelid conditioning as a function of CS intensity, UCS intensity, and manifest anxiety scale score, J. exp. Psychol.. $1963,66,429-438$.

Bolles, R. C., \& Seelbach, S. E. Punishing and reinforcing effects of noise onset and termination for different responses. $J$. comp. physiol. Psychol., 1964, 58, 127-131.

Campbell, B. A. The fractional reduction in noxious stimulation required to produce "just noticeable" learning. J. comp. physiol. Psychol., 1955, 48, 141-148.

Harrison, J. M., \& Abelson, R. M. The maintenance of behavior by termination and onset of noise. J. exp. Anal. Behav., 1959, 2. 23-42.

Gray, J. A. Relation between stimulus intensity and operant response rate as a function of discrimination training and drive. J. exp. Psychol., 1956, 69, 9-24.

Kesson, W. Response strength and conditioned stimulus intensity. J. exp. Psychol., 1953, 45, 82-86.

Myers, A. K. Effects of CS intensity and quality in avoidance conditioning. J. comp. physiol. Psychol., 1962, 55, 57-61.

Walker, E. G. Eyelid conditioning as a function of intensity of conditioned and unconditioned stimuli. J. exp. Psychol., 1960, 59, 303-31t.

Zener, K. Individual differences in the functional relationship of conditioned stimulus intensity and response magnitude. Amer. Psychologist, 1949, 4, 218. 